\title{
LIBERTAD NEGATIVA, AUTONOMÍA PERSONAL Y CONSTITUCIÓN
}

\author{
NEGATIVE LIBERTY, PERSONAL AUTONOMY AND CONSTITUTION
}

\begin{abstract}
JUAN IOSA*
RESUMEN: Inquiero sobre la existencia y límites de un principio moral de no interferencia con la vida privada, tal que requiera sanción constitucional. Evalúo diversas alternativas de conceptualización y valoración de su fundamento: la autonomía personal. Quienes nieguen su valor tendrán concepciones deflacionistas de la no interferencia. Pero valorarla no implica un único modo de concebirla. Evalúo una formulación amplia que no se compromete con ninguna concepción. Presento luego las de Frankfurt/Dworkin y Raz. Indico las consecuencias que de ellas se siguen respecto de la delimitación del ámbito de libertad negativa. Ofrezco una redacción de la garantía conforme con cada postura y la contrasto con casos problemáticos.
\end{abstract}

Palabras clave: Autonomía personal, libertad negativa, autonomía como no interferencia, perfeccionismo, liberalismo.

ABSTRACT: I inquire about the existence and limits of a moral principle of non-interference with privacy, such that should be constitutionally sanctioned. I evaluate various alternatives of conceptualization and assessment of its ground: personal autonomy. Those who refuse its value will have deflationary conceptions of noninterference. But valuing it does not involve a single mode of conceiving it. I assess a broad formulation that is not committed to any conception. Then I present Frankfurt/Dworkin's and Raz's conceptions. For all of them I highlight the consequences that follow concerning the delimitation of the scope of negative freedom, offer a wording of the warranty consistent with each posture and contrast it with hard cases.

Key words: Personal autonomy, negative liberty, autonomy as noninterference, perfectionism, liberalism.

\section{INTRODUCCIÓN}

Todas nuestras democracias constitucionales limitan de algún modo el poder del Estado sobre el individuo; establecen, en otras palabras, un ámbito de autonomía como no interferencia o libertad negativa ${ }^{1}$. Ahora bien, el modo específico de regulación de dicho ámbito varía significativamente de constitución en constitución. Algunas, como la

\footnotetext{
* Doctor en Derecho y Ciencias Sociales por la Universidad Nacional de Córdoba (UNC), Argentina; Investigador del Consejo Nacional de Investigaciones Científicas y Técnicas (CONICET) y de la Universidad Siglo 21 (US21) de Córdoba; Docente de Filosofía del Derecho de la UNC. Dirección postal: Araoz de Lamadrid 441, departamento 3, Córdoba, Argentina. Correo electrónico: juanfiosa@gmail.com. Agradezco a Gustavo de la Orden, Horacio Sánchez, Pedro Salazar, Antonio Bascuñán y Moisés Vaca la posibilidad que me brindaron de discutir estas ideas en sus universidades y otros foros académicos.

1 Aquí, por "libertad negativa" se entiende un límite normativo al poder del Estado sobre el individuo, no una mera ausencia de interferencia como cuestión de hecho. Tal como quedará aclarado más abajo, goza de libertad negativa quien tiene derecho a ser dejado solo para hacer lo que le parezca, en tanto no interfiera con los demás.
} 
Constitución argentina, establecen un principio general que contiene y del cual derivan al menos buena parte de los derechos particulares explícitos (libertad religiosa, de trabajo, de reunión, de asociación, de circulación, derecho de propiedad, a la intimidad, etc.) $)^{2}$. Otras, como las constituciones mexicana, chilena y española, solo establecen esas libertades particulares; la existencia del referido principio es algo cuya determinación queda librada a la discusión doctrinaria y a las decisiones jurisprudenciales ${ }^{3}$. En todo caso, ¿existe tal principio como cuestión de moralidad política, i.e., con independencia de lo que las constituciones particulares digan? ¿Cuál sería su fundamento? De existir parece que sería deseable que las constituciones que solo lo tienen implícito le den una formulación explícita. En ese caso ¿cómo cabría delimitarlo, i.e., qué acciones deberían quedar protegidas por este principio y qué acciones estarían más allá de su alcance? Consecuentemente ¿cómo debería redactarse el texto que lo constitucionalizara? ${ }^{4}$.

Estas preguntas también son una guía útil respecto de la correcta interpretación de una redacción específica, positiva, de la garantía. Pensemos en el artículo 19 de la Constitución Nacional Argentina. En su primer párrafo este artículo establece: "Las acciones privadas de los hombres que de ningún modo ofendan al orden y a la moral pública, ni perjudiquen a un tercero, están solo reservadas a Dios y exentas de la autoridad de los magistrados".

¿Cómo debiéramos interpretarlo? Supuesto que el modo correcto de interpretar un artículo semejante pase en parte por dar cuenta de cuál sea su mejor lectura moral, cabría

Independientemente entonces de la corrección de la impugnación republicana al concepto de libertad negativa (sobre la cuál tengo mis dudas), aquí este incluye el concepto republicano de no dominación. Agradezco a María Victoria Inostroza y Lorena Ramírez y José Luis Martí sus aportes en el sentido de la necesidad de clarificar este punto.

2 A favor de la derivación de las libertades particulares a partir de este principio general ver NiNo (2007) p. 202 .

3 Mi afirmación, por supuesto, es controvertida. La Constitución de Chile, por ejemplo, además de proteger libertades específicas en su artículo 19, en su artículo 1 inc. 1 afirma que "Las personas nacen libres e iguales en dignidad y derechos". ¿Equivale esto a un principio general como el que estoy proponiendo? Así lo entienden García Pino y Contreras Vásquez: "Un primer significado constitucional de autonomía corresponde al reconocimiento de la agencia moral de los individuos, en cuanto personas libres e iguales en dignidad y derechos. Dicha agencia moral es uno de los fundamentos del principio de dignidad, establecido en el art. 1 , inc. $1^{\circ}$ de la Constitución. Esta idea de autonomía presupone que los individuos son entes capaces de tomar sus propias decisiones para desarrollar un plan de vida, de acuerdo a sus concepciones de lo bueno. Por ello, se conecta directamente con el libre desarrollo de la personalidad. Al reconocer el carácter autónomo de los individuos, el ordenamiento constitucional mandata la tutela de los presupuestos fundantes que permiten a las personas el desarrollo de su plan de vida. Los derechos fundamentales son parte de los presupuestos de dicha autonomía, es decir, a través del ejercicio legítimo de tales derechos, los individuos pueden desarrollarse en una comunidad. Por ello, la comunidad política no puede suprimir dicha agencia moral, ya que estaría tratando a un individuo como medio y no como un fin en sí mismo. En tal sentido, la Constitución prohíbe la instrumentalización del individuo. Tal consideración sería el fundamento del denominado principio de inviolabilidad de la persona humana" García Pino y Contreras VÁzquez (2014) p. 93. Pero la pregunta subsiste. ¿Determina acaso el artículo 1 de la Constitución de Chile si el legislador ordinario puede o no prohibir la prostitución y los prostíbulos, la eutanasia voluntaria, el consumo de estupefacientes y casos semejantes? En todo caso creo que es posible lograr una redacción mucho más explícita sobre el punto.

4 La cuestión es especialmente relevante para Chile, actualmente embarcado en una discusión colectiva sobre la necesidad de redefinir su pacto constitucional. 
primero identificar el modo moralmente óptimo de concebir el principio y luego precisar la medida en que la práctica interpretativa del derecho en cuestión, en este caso el argentino, permite acercarse a esa concepción.

El presente trabajo es solo el comienzo de una investigación tendiente a retomar estas viejas preocupaciones liberales. Sostendré aquí que el ámbito de no interferencia encuentra su fundamento y alcance en el principio de autonomía personal, que este es el criterio que nos permite evaluar qué acciones deben quedar excluidas del poder normativo y coactivo del Estado5. Si este es el caso la discusión se traslada entonces a la delimitación del concepto y la determinación del valor de la autonomía personal.

\section{DisTinCIÓN ENTRE “AUTONOMÍA COMO NO INTERFERENCIA” Y “AUTONOMÍA PERSONAL"}

Para esclarecer la relación entre los conceptos de autonomía personal y autonomía como no interferencia me parece importante recordar la distinción de Feinberg entre cuatro sentidos de la palabra "autonomía" cuando se aplica a individuos: Puede ser un derecho, una capacidad, una condición actual del sujeto o un ideal del carácter ${ }^{6}$.

Con estas nociones en mente retomemos nuestra distinción central. Una persona goza de autonomía personal cuando es autora de su propia vida, decide por sí misma cómo llevar adelante su vida, lleva la vida que quiere llevar. Aquí la idea básica parece ser o bien la autonomía como estado actual o bien como ideal. De hecho, valoramos el derecho y las capacidades necesarias para la autonomía personal porque valoramos el estado en que se goza de autonomía personal o una situación ideal en que ese estado se desarrolla máximamente. Creo que esta formulación un tanto vaga basta por ahora para distinguir la autonomía personal de la autonomía como no interferencia. La primera es esencialmente positiva, se predica del individuo en tanto toma decisiones que guían su vida o se identifica con ciertos estados y acciones suyas, i.e., es una de las formas de concebir el autogobierno. Por su parte, la idea central detrás de la autonomía como no interferencia es la de un derecho del sujeto: un derecho a que otros se abstengan de determinadas acciones. El derecho del agente a no sufrir interferencia no es entonces sino un deber de abstención puesto en cabeza del Estado y los demás individuos, i.e., un deber negativo. De aquí que sea muy distinto afirmar que una persona tiene autonomía en el sentido de tener asegurado un espacio de no interferencia estatal, se lo defina como se lo defina, a afirmar que esa misma persona es autora de su propia vida. Una persona que goza de autonomía como no interferencia no necesariamente decide por sí misma qué vida lleva. Puede simplemente derivar por la vida sin tomar decisiones o actuar conforme las decisiones de otro. Pero gozar de un espacio de no interferencia es condición necesaria del ejercicio actual de la autonomía. Podemos referirnos entonces a la autonomía como no interferencia como 'derecho a la autonomía personal' y a la autonomía personal como condición actual simplemente como 'autonomía personal'.

\footnotetext{
5 La tesis, por supuesto, no es nueva. Al respecto ver por ejemplo Raz (1986) pp. 400, 410 y Nino (2007) p. 205. Pero si bien es muy trabajada en el ámbito de la filosofía política, su aplicación y desarrollo en el ámbito de la teoría constitucional es menor.

6 Feinberg (1986) p. 28.
} 
Una aclaración más. El concepto de autonomía personal como la situación de la persona que decide por sí misma el curso de su vida, que toma por sí misma las decisiones que han de moldear su destino, es relativo tanto a las decisiones autorreferentes como las que implican consecuencias respecto de las vidas ajenas. No hay nada contradictorio en decir que goza de autonomía personal aquel que decidió por sí mismo, libremente, llevar una vida de asesino serial, que tal decisión es fruto de su autonomía. Pero sobre las opciones que interfieren ilegítimamente con la vida o la libertad de terceros no tenemos, en principio, ningún inconveniente con que sean regidas por el derecho. En este sentido, tal como afirmara Rawls, lo correcto tiene prioridad sobre lo bueno ${ }^{7}$. Es por ello que las preguntas más bien abstractas realizadas más arriba están vinculadas a una preocupación muy concreta: ¿puede el Estado obligar a los ciudadanos a tomar determinadas opciones o desalentarlos de otras aun cuando dichas opciones no hagan referencia a la vida de los demás? ¿Puede el derecho imponer criterios morales autorreferentes? ¿No tenemos, al menos en lo que afecta meramente a cada uno de nosotros, derecho a hacer lo incorrecto e imprudente? ${ }^{8}$.

Luego de esta distinción conceptual cabe volver sobre la tesis sustantiva: el alcance y los límites de la autonomía como no interferencia, del derecho a la autonomía personal, han de determinarse por un lado, en función de cómo se entienda la autonomía personal y, por otro, en función de cuánto se la valore: deberá haber tanta no interferencia como sea necesaria para asegurar la adecuada satisfacción de este valor (al menos en lo que de la no interferencia ajena dependa). Gozar de un espacio de no interferencia es, en otras palabras, condición necesaria (aunque no suficiente) del ejercicio de la autonomía personal. Analizaré entonces por un lado las ideas de los detractores de la autonomía personal (teorías perfeccionistas) y por otro las ideas de sus abanderados (concepciones liberales). El criterio de distinción, aun si luego del análisis debe ser revisado, puede formularse en los siguientes términos. Hay dos cosas que se pueden considerar que hacen a nuestro bienestar: el actuar bien y el actuar en virtud de nuestras propias decisiones o identificaciones. El perfeccionismo tradicional juzga las acciones (y las vidas a las que las acciones dan forma) por la bondad de su contenido; el que ese curso de acción sea fruto de nuestra libre elección es algo que, cuanto menos, pasa a un segundo plano. El liberalismo antiperfeccionista valora la libre elección de nuestras acciones autorreferentes por sobre el contenido de esas elecciones (e incluso, en alguna de sus variantes, define la bondad de las acciones autorreferentes en tanto sean producto de nuestra libre elección). El perfeccionismo liberal, por su parte, valora tanto la bondad de la acción como el hecho de que sea producto de nuestra elección. Tiene, por lo tanto, que llegar a algún compromiso entre ambos valores. Pero claro, también cabe que el constituyente deba proteger este valor en términos abstractos, que aun valorando la autonomía deba abstenerse de cualquier compromiso con una concepción específica, dejando, por el contrario, la determinación precisa de su contenido y alcance al juez, más informado en el caso concreto.

\footnotetext{
RaWls (1971) p. 31. Esta idea ya estaba, como es sabido, en el principio de libertad de Kant enunciado en Teoría y Práctica (veáse. KANT VIII: 290).

8 La pregunta en estos términos tiene una larga tradición. Entre otros Mill (1984); STEPHEN (1991); DevLIN (1965); HART (1963); DwORKIN (2012).
} 
Presentaré primero entonces, aunque de un modo un tanto esquemático, las tesis básicas del perfeccionismo. Revisaré luego la posibilidad de constitucionalizar el derecho a la autonomía en términos de principios y conceptos abstractos (en el sentido de Ronald Dworkin). Reconstruiré por último dos concepciones de la autonomía personal centrales en el debate contemporáneo. Primero una concepción liberal bastante radical, la teoría jerárquica de la autonomía personal de Harry Frankfurt ${ }^{9}$ y Gerald Dworkin ${ }^{10}$. Distinguiré dos lecturas posibles de esa propuesta: la concepción empírica y la concepción normativa de las metapreferencias. Esbozaré luego una crítica a la viabilidad de la primera lectura, crítica que nos conducirá al análisis de la última concepción, el perfeccionismo liberal de Raz.

Previa evaluación de las razones a favor y en contra de cada alternativa, el trabajo debiera concluir con la identificación del modo correcto de concebir la autonomía personal y con una afirmación respecto de su peso normativo. Ello permitiría sostener de modo categórico determinada delimitación del ámbito de no interferencia, i.e., precisar cuáles acciones genéricas deben quedar incluidas y cuáles fuera de la potestad coercitiva del Estado. Pero tal evaluación equivaldría a dar acabada cuenta del debate entre perfeccionismo y liberalismo, algo que se encuentra más allá de mis actuales posibilidades. Me conformaré entonces con indicar la dirección que debe seguir la indagación; en este sentido mi trabajo tendrá la forma del esbozo de un paisaje teórico antes que la de un argumento acabado.

Pero aun si no puedo ofrecer aquí una evaluación categórica de las credenciales de cada teoría ni determinar cuál deba preferirse, sí indicaré una propuesta de redacción del derecho a la autonomía personal conforme con cada una de ellas. A su vez testearé las consecuencias de estas diversas propuestas de redacción de la garantía constitucional con dos casos problemáticos. Por un lado, la prohibición de la instalación de prostíbulos que ha tenido lugar en Córdoba, Argentina, como parte de una ley que declama ser una herramienta en la lucha contra la trata de personas. Dicha ley ha sido fuertemente rechazada por las trabajadoras sexuales asociadas en la Asociación de Meretrices Argentinas. En segundo lugar, la sanción penal del consumo de prostitución que forma parte de la agenda de diversos grupos activistas y es ley en los países nórdicos.

\section{PerfecCionismo y AUTONOMíA}

La concepción perfeccionista de la política afirma que no existe un principio general de moralidad política que prohíba al Estado el promover directamente el bien, aun cuando el bien esté sujeto a un desacuerdo razonable ${ }^{11}$. Para el perfeccionismo "el Estado tiene la responsabilidad y el derecho de perseguir el bien, el bienestar, el florecimiento y la excelencia de todos sus ciudadanos y desalentarlos, aun coercitivamente, de al menos algunas de las acciones y disposiciones que los dañarían, degradarían o humillarían, incluso si esas acciones y disposiciones son autorreferentes"12. Para la discusión que aquí propongo hemos de suponer que estamos hablando de lo que realmente es bueno para la vida de las perso-

\footnotetext{
FRANKFURT (1971).

10 Dworkin (1976) y Dworkin (1988).

11 WALL (2012).

12 Finnis (1987) p. 434.
} 
nas, no de lo que la gente que está en posiciones de poder o la sociedad en general piensa que es bueno. Hemos de partir entonces de lo bueno según una moral crítica y no según una moral positiva ${ }^{13}$. Supuesto esto, cabría distinguir dos tesis típicas del perfeccionismo. Primero, lo que es bueno para un individuo o lo que satisface sus intereses (objetivos) es independiente de sus propios deseos e identificaciones, de su elección de forma de vida, i.e., no es definido autónomamente. Segundo, a consecuencia de lo anterior el Estado puede, incluso a través de la fuerza, dar preferencia a aquellos intereses y planes de vida que son objetivamente mejores. Consecuentemente, aun si una acción es privada en el sentido de no interferir con terceros, el hecho de que implique autodegradación moral constituye una razón suficiente para que el derecho la obstaculice, induciendo así a los hombres y mujeres a adoptar modelos de conducta digna ${ }^{14}$. En Argentina Valiente Noailles ha sostenido esta doctrina como fundamento de su interpretación de la norma constitucional: "Para proteger la moral pública el Estado no solo debe evitar -a través de una legislación adecuada que contemple tanto la persuasión como el ejercicio de la policía de la moralidad- los ataques contra ella, sino que debe contribuir a elevar el grado de moralidad de la población a través de los medios directos a su alcance, que sean compatibles con nuestro orden constitucional" 15 .

Aun cuando el perfeccionismo sea fundamento corriente de gobiernos autoritarios, no debemos creer que esté esencialmente vinculado al autoritarismo ni que deba desecharse como teoría política plausible. Por el contrario, goza de renovada vitalidad y hoy es la opción teórico-política al liberalismo ${ }^{16}$. Después de todo, ¿para qué queremos el Estado sino porque creemos que es una herramienta que nos puede ayudar a vivir mejor?

Pero también es claro por qué las concepciones perfeccionistas tienden a minimizar el alcance y en última instancia a descartar la existencia de un principio general de no interferencia del Estado con el individuo. Si lo bueno para una persona es definido objetivamente, sin referencia a sus decisiones, no se ve por qué el Estado debiera autolimitarse y dejar de promover el bien de ese individuo en el ámbito de sus decisiones autorreferentes. Si en ese ámbito elige mal y se equivoca, no hay ninguna razón para no traerlo nuevamente a la buena senda, incluso coactivamente. No habría, en otras palabras, ningún fundamento normativo sólido para tratar de modo diferente los deberes para con uno mismo y los deberes para con los demás. O más aun, no tendría sentido la distinción.

Es por ello que si el perfeccionismo es la teoría política correcta entonces no cabrá establecer en nuestras constituciones ningún principio general de protección de la autonomía personal. Bastará con establecer libertades particulares que protejan estados, acciones y decisiones valiosas según el contenido de la doctrina perfeccionista correcta. Entre ellas

\footnotetext{
13 Nuestro objeto de análisis es el perfeccionismo, no el moralismo legal. Esta última es una doctrina sobre la justificación de la imposición coercitiva de la moral positiva, las creencias morales sostenidas por la mayoría de la sociedad, incluidas las relativas a acciones autorreferentes. Afirma que tal imposición está justificada independientemente de si esas creencias encuentran adecuado respaldo en razones o se sostienen luego de la reflexión.

14 Nino (2007) c. V y X.

15 Valiente Noailles (1966) p. 34.

16 Cfr. Véase Raz (1986); HurKa (1993); HaKSAR (1979); Wall (1998).
} 
cabe esperar que figuren la mayoría de los derechos que pueblan nuestras constituciones: de propiedad, de trabajar, de expresión, de reunión, etc., pero no un principio general del cual estos se deriven.

El perfeccionismo, como toda doctrina que se precie de tal, es pasible de un sinnúmero de objeciones. Entre otras deficiencias podemos imputarle insuficiente valoración de nuestra capacidad de elección y decisión y una injustificada creencia en la mayor capacidad de las autoridades de reconocer lo que es bueno para los súbditos. Pero evaluar las posibles objeciones no viene aquí al caso. Sí corresponde revisar las consecuencias de esta doctrina para nuestros casos testigo: la prohibición de la instalación de prostíbulos por un lado y de la oferta y demanda de sexo, por otro. Supongamos que acordamos que es inmoral, que no hace bien consumir prostitución, aun frente a una persona que consiente. Que también es inmoral ofrecer sexo a cambio de dinero: la persona que así actúa, es posible argumentar, no se respeta adecuadamente a sí misma en tanto, en nuestras sociedades al menos, la sexualidad es parte central de la subjetividad y por lo tanto el cuidado de sí requiere que la vida sexual no ingrese al comercio (un perfeccionista podría decir que estas acciones son contrarias al perfeccionamiento del ser). Pues bien, supuesto que hay buenas razones morales para no consumir ni ofrecer sexo a cambio de dinero, el perfeccionista no ve por qué no se habrían de inhibir coactivamente estas conductas. El consentimiento de los involucrados no cambia en absoluto la calificación moral de las conductas, el hecho de que realizarlas es algo que nos degrada. Por lo tanto no es óbice a su prohibición estatal. El Estado tendría el derecho moral de sancionar la prostitución, el proxenetismo y el consumo.

\section{LibERALISMO Y AUTONOMÍA}

La doctrina liberal ha evolucionado desde sus defensores clásicos (Locke, Kant, Mill, ) a los contemporáneos (Dworkin, Rawls, Nino y muchos otros). En todo caso, si hay algo que comparten estos autores es la creencia en que toda restricción a la libertad individual debe estar justificada ${ }^{17}$. Un gobierno es legítimo entonces solo si respeta ciertos límites en relación a los individuos sometidos a su poder. Particularmente el liberalismo entiende que el gobierno no está para promover el bien de las personas (especialmente no si ello implica ir más allá de su consentimiento) sino para evitar que estas, al perseguir su propio bien en los términos que ellas mismas lo conciben, se interfieran mutuamente ${ }^{18}$. Este principio de neutralidad estatal implica, como puede verse, una clara distinción entre el ámbito público, donde hay espacio para la intervención legítima del poder estatal, y el ámbito privado en que las personas persiguen su propio bien. En este último, en tanto esa búsqueda no afecte a terceros, dicha intervención está proscripta.

En el ámbito privado entonces rige el principio de autonomía como no interferencia. Nino lo formula en los siguientes términos: "Siendo valiosa la libre elección individual de planes de vida y la adopción de ideales de excelencia humana, el Estado (y los demás indi-

\footnotetext{
17 Gaus (2011).

18 Quong (2011) p. 1. Recordemos que para el liberalismo los intereses y fines de los individuos son anteriores al Estado. El Estado justamente surge para evitar que los individuos, al perseguir cada uno su propio interés, se obstruyan y dañen mutuamente. Si este es el caso, mal podría el Estado imponerles intereses y fines.
} 
viduos) no debe interferir en esa elección o adopción, limitándose a diseñar instituciones que faciliten la persecución individual de esos planes de vida y la satisfacción de los ideales de virtud que cada uno sustente e impidiendo la interferencia mutua en el curso de tal persecución"19.

Para el liberalismo entonces, en tanto no dañen a terceros, las acciones y estados que sean fruto de la identificación del agente con un modo de vida (i.e., en tanto esa vida sea de su elección, en tanto él o ella pueda considerarse su autor o autora), están exentos de la autoridad de los magistrados: no hay ninguna razón que pueda justificar la injerencia estatal. Pero hay diversos modos de concebir lo que significa que una persona sea autora de su vida y diversas redacciones posibles para un principio protector de ese valor. Veamos primero una redacción suficientemente amplia que, valorando la autonomía, no se compromete con ninguna concepción específica.

\section{a) Derecho a la autonomía como principio/concepto}

Suponiendo entonces que valoramos la autonomía, nos preguntemos ahora si la mejor versión de un artículo que constitucionalizara ese derecho podría ser una que redacte la garantía en términos muy abstractos, tales que no evidencien un compromiso estricto con ninguna justificación específica ni delimiten muy claramente los casos incluidos en su alcance sino que dejen esta tarea a los jueces. Una redacción semejante invitaría a pensar el estándar en cuestión en términos de principios antes que de normas, de conceptos antes que de concepciones, en el sentido de Ronald Dworkin.

No tengo en claro si los textos constitucionales tienen algún rasgo que hace normativamente necesario que el legislador redacte al menos sus cláusulas dogmáticas como conceptos antes que como concepciones. Tampoco si el aplicador debe interpretarlas como si el legislador las hubiese redactado en esos términos o si esto es un rasgo contingente, dependiente del modo en que el constituyente haya decidido redactar el texto. Pero me resulta convincente la argumentación de Atria ${ }^{20}$ en el sentido de que esto es deseable, que si los derechos fundamentales han de cumplir una función constituyente de la comunidad política, de un nosotros que se reconoce como tal justamente por referencia a esos textos, entonces ellos deben estar redactados e interpretados en términos de conceptos y no de concepciones. Veamos entonces la propuesta de Dworkin en este sentido.

Para Dworkin las normas, cuando son válidas y aplicables a los hechos del caso, determinan la solución. Los principios, por el contrario, cuentan a favor de determinada solución, inciden en ese sentido, pero no necesariamente la determinan. En segundo lugar los principios tienen una dimensión de peso carente en las normas, es decir, cuando hay un conflicto de principios la cuestión se resuelve por el peso relativo de los principios mientras que los conflictos normativos se resuelven por un estándar externo a las normas en conflicto tal como lex superior, posterior, etc. ${ }^{21}$. Es debatida cuál sea la mejor forma de dar

\footnotetext{
19 NinO (2007) p. 204. Como puede verse, Nino combina en un solo principio las dos ideas (libertad negativa y autonomía personal) que aquí hemos tratado de deslindar.

20 Atria (2016) pp. 274-276.

21 DwOrKIn (1993) pp. 75-78.
} 
cuenta de esta distinción. Particularmente me gusta la idea de ver en los principios razones de primer orden y en las normas razones protegidas, en el sentido de $\mathrm{Raz}^{22}$. Las razones de primer orden son razones a favor o en contra de realizar determinada acción. Las razones protegidas son una combinación sistemática de una razón de primer orden para realizar determinada acción (la ordenada por la norma) y una razón excluyente, de segundo orden, para no tener en cuenta las razones en contra de realizar esa acción. Cuando el constituyente legisla mediante principios está diciendo que hay buenas razones (de primer orden) para seguir determinado curso de acción, está, en otras palabras, consagrado constitucionalmente un valor, por ejemplo la libertad de expresión. Pero este valor puede entrar en conflicto, y eventualmente ceder, frente a otro valor, por ejemplo, el derecho a la intimidad. De aquí que un principio anuncie una ponderación. Cuando dicta una norma, en cambio, el legislador ya ha realizado el balance de razones de primer orden y decidido qué razón tiene mayor peso, excluyendo las demás. En este caso el juez no tiene que hacer ningún balance ${ }^{23}$.

Veamos ahora a la distinción entre concepto y concepción. Según Dworkin tiene que ver con un contraste entre niveles de abstracción en los que puede ser estudiado determinado objeto de interpretación, un texto, una práctica, etc. ${ }^{24}$. Parafraseando a Dworkin ${ }^{25}$ supongamos que un grupo comparte la idea de que las decisiones, los actos y las vidas de las personas pueden gozar o carecer de una propiedad moral que ellos llaman autonomía personal, que consiste en que las personas que la poseen deciden por sí mismas sus acciones y en general el curso de sus vidas, son, en buena medida, sus autoras, mientras que las vidas que carecen de esta propiedad son principalmente producto de la voluntad de alguien que no es su titular. Gracias a ello están de acuerdo en muchos casos de autonomía y en muchos de falta de autonomía; también lo están respecto de calificar a ciertos actos, propios o ajenos, como dañosos para la autonomía de una persona. En este caso el grupo tiene un concepto de autonomía personal. Pero, más allá de este núcleo de consenso, el grupo puede disentir respecto de otros casos de modo tal que un observador podría pensar que tienen diferentes teorías de bajo qué condiciones una determinada acción es consistente o contradictoria con la autonomía. Es decir, pueden diferir respecto de los principios más fundamentales que justifican calificar de respetuosa de la autonomía a determinada acción. En este caso los miembros del grupo tienen diversas concepciones de la autonomía.

Ahora bien, al dar instrucciones o directivas relativas a la autonomía los legisladores de esta comunidad pueden o bien apelar a conceptos o bien a concepciones. Si hacen lo primero entonces delegan en los jueces la responsabilidad de desarrollar y aplicar su propia concepción de la autonomía para los casos controvertidos. Y las concepciones son defendibles sobre la base de razones de modo que se supone que hay concepciones mejores que otras. El punto es que quien legisla mediante conceptos está dispuesto a admitir que un caso que según su propia y contingente concepción quedaba dentro del alcance de su instrucción en realidad no lo estaba, en tanto alguien le muestre una concepción supera-

\footnotetext{
RAZ (1991) p. 238.

23 En este sentido Atria (2012).

24 DwOrkin (1986) pp. 70-71.

25 DWORKIN (1993) pp. 214-215.
} 
dora que lo excluya. Por lo tanto cuando cambia su teoría, su concepción, no cambia su estándar. Si por el contrario, el legislador dictó la instrucción con ánimo de formular determinada concepción de la autonomía, si quiso que la cuestión se regule de acuerdo a los principios fundamentales que tenía en mente y que no abarque más ni menos casos que los alcanzados por esos principios, el aplicador deber atenerse a esa concepción, no hay nada delegado a su discernimiento.

A mi entender un poder constituyente que quisiera establecer el derecho a la autonomía personal como un principio antes que como una norma, como un concepto antes que como una concepción, debería dictar una directiva del siguiente tono:

Esta constitución garantiza el derecho a la autonomía personal de los ciudadanos en tanto su ejercicio no afecte un ejercicio equivalente del mismo derecho por parte de terceros ${ }^{26}$.

Un texto de este tipo afirma que existe un derecho moral a gozar de un espacio de no interferencia y lo constitucionaliza. Un derecho moral es, de nuevo siguiendo a Dworkin, un triunfo contra las mayorías, un derecho contra el Estado. De modo que si el Estado reconoce este derecho no podrá dejarlo de lado sobre la base de una ley votada por mayorías circunstanciales. Pero un derecho moral redactado en estos términos tiene al menos otras dos propiedades que debemos atender. Primero, puede ceder frente al derecho moral de un tercero, un derecho concurrente. Segundo, está redactado en términos tales que su contenido específico, su alcance, debe ser determinado por el juez sobre la base de la específica concepción de la autonomía personal que considere correcta. Esto tiene consecuencias tanto positivas como negativas.

Por un lado nos permite dar cuenta de nuestras intuiciones contrarias a una definición muy estricta del derecho a la autonomía personal tal que si alguna acción es una instancia de ejercicio de la autonomía y no afecta la autonomía de terceros entonces inmediatamente está protegida y es inatacable. Estoy pensando en casos como los contratos Shylock y las exhibiciones obscenas.

En los contratos Shylock una persona vende sus órganos o contrae una deuda con otra bajo la condición de que si no paga en tiempo entonces deberá entregarle un órgano u otra parte de su cuerpo. Este contrato puede ser firmado en condiciones de autonomía plena, como sucede en "El mercader de Venecia". Una lectura estricta del artículo 19 de la Constitución argentina, como si estipulara una norma bajo una concepción liberal radical (véase más adelante) llevaría a declararlo válido. Sin embargo contratos de este tipo, aun si firmados autónomamente y respetuosos del orden y la moral pública en el sentido del referido artículo bajo esa concepción (i.e., los deberes para con los demás), nos parecen inmo-

\footnotetext{
26 Recordemos que la pregunta que nos estamos haciendo (cuál sea la mejor redacción posible del estándar que constitucionalizara el derecho a la autonomía personal) bien puede ser una guía apropiada para preguntarse por la mejor lectura moral de una redacción concreta de esa garantía. Pensemos en el artículo 19 de la Constitución argentina ¿cómo debiéramos interpretarlo? ¿Como un principio o como una norma? ¿Como expresando el concepto de derecho a la autonomía personal o, por el contrario, como refiriendo a una concepción específica? Si la propuesta que estamos analizando fuera la mejor desde el punto de vista moral, ello sería un argumento muy fuerte (aunque no concluyente, pues hay otras dimensiones como su ajuste a la práctica institucional) para interpretar en sus términos este artículo.
} 
rales en un sentido más amplio, independiente del consentimiento del afectado; de hecho creo que los jueces argentinos los declararían inválidos por esa razón. Por el contrario, si concebimos el estándar como un principio, bien puede el juez decir que aquí hay otro principio en juego, un derecho concurrente, el derecho a la integridad corporal por ejemplo, y fallar sin mayor problema en contra de la validez del contrato. Y ello sin negar que, prima facie, quedaba protegido por la garantía.

El caso de las exhibiciones obscenas, supuesto que nuestras intuiciones morales sean favorables a su punición, nos permite pasar de las ventajas de legislar este derecho en términos de principios a las ventajas de hacerlo en términos de conceptos. Supongamos que el artículo 19 de la Constitución argentina deba ser leído como expresión de una concepción tal que afirma que solo puede sancionarse una acción cuando causa daño a otro y a su vez define 'daño' en un sentido muy estricto, tal que no incluye las ofensas al sentido moral, al pudor de los demás, como buena parte de la doctrina liberal sostiene que debe ser el caso si la noción de daño ha de funcionar como límite al poder coercitivo del Estado y de las mayorías morales. Pues bien, así las cosas, la penalización de las exhibiciones obscenas es inconstitucional. $\mathrm{Si}$, en cambio, entendemos que el artículo impone un concepto de autonomía personal, el juez tiene espacio para desarrollar la concepción que considere correcta, una que, por ejemplo, conciba las ofensas como daño, de modo tal que le permita excluir las exhibiciones obscenas del alcance de la garantía.

Pero estas conclusiones también pueden ser leídas como razones en contra de redactar la garantía en términos de principios y conceptos en lugar de normas y concepciones. Pues al estar redactada como un principio y no como una norma, aun de aceptar que el caso queda protegido por el principio, eso no nos asegura una decisión favorable. Como en los contratos Shylock, puede haber otro principio que en el caso tenga mayor peso y que incline la balanza en contra de su protección. Y al estar redactada en términos de conceptos, no podremos determinar por la mera lectura del texto constitucional si determinada acción está protegida o no por la garantía. En este sentido el texto deja de instaurar una garantía en sentido estricto: no sabremos antes de la decisión del juez si el acto en cuestión está o no incluido en su alcance. Específicamente, deberemos esperar a conocer cuál es la concepción que el juez considera correcta, i.e., que se expida sobre la validez de la norma dictada por el legislador infraconstitucional (la que prohíbe, por ejemplo, las exhibiciones obscenas).

De hecho esto es lo que sucederá con nuestros casos testigo: la prohibición de los prostíbulos y del consumo de prostitución. Respecto de este último, por ejemplo, tanto el legislador como el juez podrían tener una concepción tan exigente del consentimiento requerido para que una decisión cuente como propia, como autónoma, tal que nunca pueda haber ejercicio consentido de la prostitución. De aquí que la garantía redactada en los términos arriba sugeridos no serviría como límite a la sanción legal y judicial de su ejercicio ni su consumo. El problema es que no nos enteraríamos de su alcance hasta la decisión del juez. Esto por supuesto, amenaza la autonomía personal, justamente el valor que se declama proteger. Pues si no podemos saber ex ante cómo debe decidir el juez, no podemos planificar y decidir nuestras acciones teniendo en cuenta sus consecuencias ${ }^{27}$.

27 BAYÓN (1996) p. 42. 
Este no es en absoluto un argumento concluyente en contra de redactar la garantía en términos amplios. Pero sí indica que deben balancearse sus costos y beneficios contra los que tendría una redacción más estricta. Pasemos entonces al análisis y evaluación de las concepciones particulares de la autonomía personal que podrían justificar tal redacción. Detengámonos primero en una concepción mínimamente exigente (que por ello permite calificar como autónomos una muy amplia gama de planes de vida). Me refiero a lo que describiré como concepción empírica de las metapreferencias, una lectura posible de las tesis de Harry Frankfurt y Gerald Dworkin. Esta concepción es plenamente compatible con la formulación amplia del espacio de no interferencia propia del liberalismo. Cuestión aparte es si es sustentable.

\section{b) La teoría jerárquica de la autonomía: Frankfurt/Dworkin}

Las personas gozan de autonomía personal si eligen por sí mismas su plan de vida. Una forma de dar cuenta de esta idea es la siguiente. Un sujeto autónomo es aquel "guiado por consideraciones, deseos, condiciones y características que no le son impuestas externamente, sino que son parte de lo que puede considerar como (su) propio y auténtico yo" 28 . La noción de deseo auténtico está vinculada a la distinción, introducida por Frankfurt y Dworkin, entre deseos de primer y segundo orden. Un deseo auténtico es aquel con el que el yo se identifica, i.e., un deseo que el agente quiere (según un deseo de segundo orden) tener. De lo contrario su deseo es inauténtico, en cierta forma no es suyo. Así, puede tener el deseo de primer orden de fumar y el deseo de segundo orden de no ser un fumador: desear no desear fumar. Ahora bien, no se trata solo de que el yo hoy puede identificarse o no con un deseo actual. También puede identificarse o rechazar sus deseos e intereses (i.e., deseos a largo plazo) futuros. En tanto la persona puede definirse, al menos en parte, como un haz de deseos e intereses proyectados en el tiempo, podemos considerar que goza de autonomía personal aquel que haciendo uso de su capacidad de considerar y proyectar qué clase de persona quiere ser, elige por sí mismo el propio plan de vida i.e., se identifica con algunos de sus deseos e intereses futuros ${ }^{29}$.

Haré foco aquí en las ideas de Dworkin donde el problema que quiero presentar está más claro. Mostraré que hay dos lecturas posibles de sus ideas: la concepción empírica y la concepción normativa de las metapreferencias. Argumentaré que la concepción empírica, compatible con un liberalismo radical en el sentido de una prohibición absoluta de la interferencia estatal en la elección autónoma de planes de vida, debe ser rechazada. Por el contrario, hay buenas razones para decantarse por la concepción normativa ${ }^{30}$. A su vez esta última apunta en la dirección de un liberalismo perfeccionista como el de Raz.

28 Christman (2011) p. 2.

29 LAPORTA (2007) pp. 31-32.

30 La concepción empírica es en buena medida equivalente a lo que Nino denomina enfoque externo de las preferencias como meros hechos psicológicos. La concepción normativa lo es respecto del enfoque interno, en el que lo que se prefiere, se prefiere sobre la base de razones en las que se cree y que se está dispuesto a abandonar cuando se muestren equivocadas. Aquí ofrezco un argumento, espero más preciso que el de Nino, para la misma conclusión: la concepción empírica, el enfoque externo en sus términos, es autofrustrante. Al respecto ver NinO (2007) pp. 211-219. 


\section{3.b.1. Concepción empirica de las metapreferencias:}

Hay dos elementos centrales en la teoría jerárquica de la autonomía de G. Dworkin tal como aparecía en sus primeras versiones ${ }^{31}$. Por un lado, la relación entre deseos de primer y segundo orden, que cuando es de identificación es uno de los elementos de la autonomía. Por otro, la independencia procesal idea referida al modo en que se ha llegado a tener la relación entre deseos de diferente orden que se tiene, i.e., a cómo hemos llegado a identificarnos con nuestras consideraciones de primer orden o cómo hemos llegado a aborrecerlas. Al respecto Dworkin afirma que "...la identificación con sus motivaciones, o la elección del tipo de persona que quiere ser, pueden ser resultado de la manipulación, el engaño, el ocultamiento de información relevante o cosas semejantes. El agente puede haberse visto influenciado de modo decisivo por otros de modo tal que no estemos dispuestos a pensar de ellas como fruto de su propia elección" ${ }^{32}$. Si la persona se identifica con sus preferencias y no hubo influencias indebidas entonces es autónoma. Si estas influencias tuvieron lugar, aun cuando se identifique, no lo es.

Consecuentemente, sobre la base de que la autonomía se ubica al nivel de las consideraciones de segundo orden, Dworkin nos ofrece la fórmula que sirve de base a su análisis: autonomía $=$ autenticidad + independencia procesal.

Centrémonos en la idea de independencia procesal. ¿Cómo debemos entenderla? Analicemos esta idea de Dworkin: "La autonomía no puede ubicarse en el nivel de las consideraciones de primer orden, sino en el de las consideraciones de segundo orden. Aun si el hombre autónomo no puede adoptar sus motivaciones de cero, bien puede juzgarlas cuando las tiene. El individuo autónomo es capaz de dar un paso atrás y formular una actitud hacia los factores que influyen en su comportamiento"33.

Como puede deducirse de la lectura de este párrafo, respecto de aquello que resguarda la independencia procesal, no está claro si Dworkin se refiere a la posibilidad del agente de hacer un juicio en sentido estricto, es decir una evaluación del valor de sus consideraciones de primer orden (en este caso las metapreferencias serán producto antes que condición de posibilidad de la reflexión) o si lo que importa es que el individuo autónomo sea capaz de tener una actitud de segundo orden sobre sus consideraciones (básicamente creencias y deseos) de primer orden. Por supuesto, podemos pensar que una actitud de segundo orden supone -y está determinada por- un juicio de segundo orden (un juicio, ie., una creencia, sobre qué tipo de creencias y deseos es deseable tener). $\mathrm{O}$ a la inversa, podemos reducir la idea de juicio a la idea de actitud, de preferencia. Así, mi preferencia de segundo orden (preferencia que simplemente me puede suceder tener, sin que la haya elegido ni que sea producto de una evaluación) puede servirme como criterio con el cual juzgar mis prefe-

\footnotetext{
31 Posteriormente Dworkin abandonó el elemento 'identificación' como parte del concepto de autonomía personal. Pasó a sostener que lo que importa no es la identificación sino la capacidad de identificarse, es decir, la capacidad de reflexionar acerca de con cuál de mis deseos he de identificarme. Esto apunta, nuevamente, en dirección a una concepción normativa de las metapreferencias. Sobre el punto ver DwORKIN (1988) p. 15. Pero claro, como bien señala Raz, la capacidad de identificarse no es autonomía sino una de las condiciones de la autonomía.

32 Dworkin (1976) p. 25.

33 Dworkin (1976) p. 24.
} 
rencias de primer orden (aquí por 'juzgar' ha de entenderse 'prefiero o me identifico con el deseo X y no con el deseo Y'). La opción dependerá, por supuesto, de nuestras posiciones metaéticas. Pero si mantenemos separadas las ideas de actitud y de juicio, mientras la actitud de segundo orden no supone más que un estado interno del sujeto, un hecho psicológico en términos de Nino, el juicio parece suponer, tal como argumentaré más adelante, un criterio objetivo, racional, como condición de su realización.

Preguntemos entonces nuevamente. ¿Qué resguarda la independencia procesal? ¿La formación de juicios o de actitudes? Supongamos que nos decantamos por la idea de actitud. Lo que importa sería que el agente forme sus propias actitudes hacia sus preferencias de primer orden. ¿Pero cómo se forma esa actitud si no depende de un juicio en sentido estricto? Habría que asumir una de dos posibilidades: $\mathrm{O}$ bien tenemos al mismo tiempo deseos de diferente nivel (el deseo de fumar y el deseo de no desear fumar) sin relaciones causales recíprocas sino dependientes de otro fenómeno. O bien se dan relaciones causales en un sentido u en otro (desde las preferencias hacia las metapreferencias o viceversa). Cualquiera sea el caso, si nuestras metapreferencias son producto de relaciones causales no habrá razón para preferir unas a otras ni para preferir ciertos procesos causales de formación de metapreferencias por sobre otros (para ello deberíamos contar con un tercer criterio que a su vez no sea producto de relaciones causales). Ahora bien, quien haya llegado a tener una metapreferencia, tendrá necesariamente una actitud hacia sus preferencias de orden más bajo: ambas ideas están conceptualmente vinculadas. Pero una metapreferencia, salvo por su nivel, tiene exactamente la misma naturaleza que una preferencia: ambas son hechos. A su vez, si entendemos la idea de metapreferencia como un hecho, entonces parece que la idea independencia procedimental carece de sentido, se puede prescindir de ella. Pues no hay procesos causales preferibles por sobre otros si no hay criterios externos que nos permitan valorar unos por sobre otros. Así, todo el espacio de la autonomía estaría ocupado por la idea de autenticidad o identificación: soy autónomo cuando tengo los deseos (de primer orden) que deseo (en el segundo orden) tener. Llamemos a esta 'concepción empírica de las metapreferencias'.

Si esto es así, entonces todo sujeto que se identifique con sus deseos de primer orden, cualquiera sea su contenido, es autónomo y debe ser respetado en sus elecciones (aunque es difícil justificar la razón de este deber de respeto). Congruentemente con esta lectura, la autonomía personal suele pensarse como una concepción formal e inmanente del valor: son valiosos y respetables los planes de vida de las personas en tanto estas los hayan elegido libremente, ie., se identifiquen con ellos, independientemente de su contenido. De aquí el carácter radicalmente antiperfeccionista de las construcciones basadas en esta concepción de la autonomía, construcciones que prescriben que el Estado nunca debe interferir en la libre elección individual de planes de vida, cualquiera sea su contenido. De ser este el caso, la autonomía personal podría pensarse como una teoría equivalente en peso normativo a la autonomía moral, ie., como una teoría de la fuente de la normatividad (el valor de los planes de vida dependería meramente de que los hayamos elegido, ie., nos identifiquemos con ellos, independientemente de su contenido y del modo en que dicha elección o identificación haya tenido lugar). 
El análisis de Dworkin del caso del adicto a las drogas da una clave de lectura de su teoría en el sentido de la concepción empírica aquí sugerida.

"¿Qué pasa con el hombre que es un adicto a las drogas, que no puede renunciar a su compulsión por la droga pero que, sin embargo, quiere estar en las garras de su compulsión? En mi opinión, este agente es autónomo. Él, como el esclavo, no es libre, ya que tomará la droga independientemente de si desea ser motivado de esta manera. Pero, importante como este hecho es, hay otro hecho que también es cierto y también es importante. El hecho de que se identifica con su adicción. Y es esta identificación lo que estoy designando como autenticidad" 34 .

¿Cómo debería redactarse el principio constitucional si fuera cierta la teoría de la autonomía personal a la que da cuerpo la concepción empírica de las metapreferencias? Pues se debería imponer un límite muy estricto a la intromisión del Estado en las acciones autorreferentes, fruto de la identificación del agente con su plan de vida. Una posibilidad sería la siguiente: No son susceptibles de sanción jurídica las acciones y omisiones que no dañen a terceros.

O tal vez, para evitar el problemático concepto de daño: Son facultativas las acciones y omisiones que no interfieran con la autonomía personal de terceros ${ }^{35}$.

Revisemos cómo se llevan nuestros ejemplos con esta concepción de la autonomía personal como autenticidad. Sin dudas hay (o puede haber) prostitutas que se ven a sí mismas como tales, que no se sienten enajenadas y que frente a otros trabajos en igualdad de condiciones prefieren el ejercicio de la prostitución. Estas prostitutas son autónomas en este sentido, no solo que consienten cada acto de prostitución sino que, más generalmente, se identifican con su actividad y la ven como un modo adecuado de ganarse la vida. Igualmente sucede con los consumidores de prostitución. Muchos consienten cada acto y además no tienen ningún problema con verse a sí mismos como frecuentadores habituales de estos servicios. Se identifican con esta actividad y bien podemos decir que, en este sentido, son autónomos. Si son autónomos, estos planes de vida merecen respeto: el hecho de que estas personas los hayan elegido es lo que les confiere valor, no hay ningún criterio externo que pueda negarlo. El Estado debe evitar entrometerse en la persecución individual de estos planes y bien haría en legislar un principio general que prohíba semejante intromisión.

Pero claro, esta lectura solo es viable si podemos reducir la idea de reflexión a la de "tener una actitud" hacia las preferencias de nivel inferior. Pero entonces la formación de deseos de segundo orden no será producto de la reflexión: que haya coincidencia o no entre nuestros deseos de diverso orden será un hecho que nos sucede, no una situación producto de un esfuerzo derivado de una toma de conciencia sobre la naturaleza de nuestros deseos.

\footnotetext{
34 DwOrkin (1976) p. 25.

35 Obsérvese la diferencia entre esta formulación del estándar y la primera. En aquella estamos ante un principio: el artículo garantiza el adecuado respeto a la autonomía personal, pero no impone un resultado: bien puede que el derecho a la autonomía seda frente a un derecho concurrente. Aquí estamos ante una norma: si la acción no interfiere con la autonomía de terceros entonces es facultativa. El resultado se impone.
} 


\section{CONCEPCIÓN NORMATIVA DE LAS METAPREFERENCIAS}

Contra la concepción empírica, la concepción normativa de las metapreferencias concibe los deseos de segundo orden como producto de la reflexión; afirma que esta es la lectura correcta de la idea de autonomía personal en las teorías de Frankfurt y Dworkin. Ahora bien, si aceptamos que no se puede concebir la idea de autonomía personal sin acudir a la idea de reflexión caben dos observaciones. Primero, debemos preguntarnos sobre qué versará esta reflexión. Y todo parece indicar que lo que buscará determinar es qué tan deseables son nuestros deseos. Pero no parece posible reflexionar sobre la deseabilidad de nuestros deseos sin contar con criterios con los cuales evaluarlos. Si aceptamos que esos criterios son necesarios entonces podemos preguntarnos cuál es su fuente. Si no son subjetivos (recordemos que las preferencias son el objeto de la evaluación y en esta lectura las metapreferencias son el producto de la reflexión por lo que no pueden funcionar como criterios ex ante) entonces o bien nos vienen dados en virtud de la existencia de un orden normativo independiente (y se asume un compromiso con doctrinas como el iusnaturalismo o realismo moral cuyo vínculo con el perfeccionismo no es incidental) o bien emanan de nuestra autonomía moral en tanto que sujetos racionales. En este último caso parece que no podemos despegar la idea de un sujeto reflexivo de la de uno moralmente autónomo. Ahora bien, si no se goza de autonomía personal sin ejercer la reflexión entonces el Estado tiene el derecho y el deber tanto de obstaculizar aquellos planes de vida que tiendan a horadar nuestra capacidad de reflexión así como de favorecer la elección de aquellos planes que nos vuelvan más reflexivos. En este sentido apunta el perfeccionismo liberal de Raz.

\section{LiberTAD NEGATIVA Y AUTONOMÍA PERSONAL EN EL PERFECCIONISMO LIBERAL DE RAZ}

Según Raz la persona autónoma es en parte autora de su propia vida. El ideal de la autonomía personal es la visión de las personas controlando, en algún grado, su propio destino, dándole forma mediante decisiones sucesivas a lo largo de sus vidas ${ }^{36}$. Se opone a una vida de elecciones tomadas bajo coerción. También a una vida sin elecciones, o de mero derivar por la vida sin ejercitar la propia capacidad de elección ${ }^{37}$.

Para llevar una vida autónoma es preciso que se den ciertas condiciones cuyo valor es derivado del valor de una vida autónoma, llamémosle con Raz 'condiciones de la autonomía'. Son $\operatorname{tres}^{38}$ :

Apropiadas capacidades. Entre las diversas capacidades funcionales a la autonomía destaquemos aquí las mentales. Por un lado la persona autónoma goza de entendimiento: capacidad de evaluar y ponderar el valor relativo de diferentes fines, ie., estados futuros valiosos, de comprender y valorar su situación y el peso que sus decisiones tendrán en su vida y en las de los demás, de concebir los medios adecuados para llevar a cabo sus propósitos, etc. Goza, en otras palabras de capacidad de reflexionar sobre cuál de los posibles cursos de

\footnotetext{
36 RAZ (1986) p. 369.

37 Raz (1986) p. 371.

38 RAZ (1986) pp. 372-3.
} 
acción y, más genéricamente, modos de vida a su alcance, resulta preferible. Además tiene voluntad, capacidad de tomar decisiones y actuar en consecuencia ${ }^{39}$.

Adecuado rango y variedad de opciones (pues quien tiene muy restringidas las opciones disponibles no puede elegir en sentido estricto) ${ }^{40}$. Además al menos algunas opciones disponibles deben ser moralmente valiosas, pues quien tiene solo malas opciones a su alcance no goza de autonomía ${ }^{41}$.

Independencia: el ejercicio de su capacidad de opción debe ser libre de coerción y manipulación ajena. La coerción y la manipulación sujetan la voluntad de una persona a la de otra y, en este sentido, invaden su autonomía ${ }^{42}$.

Al poner en ejercicio las condiciones de la autonomía, es decir, al adoptar fines y proyectos, al comprometernos en relaciones y vínculos, damos progresivamente forma a nuestra propia vida. Alguien que decide por ejemplo estudiar una carrera, luego de tomar la decisión tiene razones para comportarse de modo de alcanzar ese fin: recibirse. Y puede actuar de modo incompatible con ese fin, fracasando por ello en su obtención de un modo que le resultaba imposible antes de adquirirlo. Es así que dotamos de valor a nuestra vida, de valores con que nuestra vida no contaba antes de esas decisiones e identificaciones. Creamos, en otras palabras, razones independientes de las razones que teníamos para asumir esos compromisos, fines y proyectos. Es así que nuestra vida se vuelve en parte nuestra propia creación: los valores y razones que, entre otras cosas, le dan forma son en alguna medida fruto de nuestros propios actos de creación normativa. En tanto que la autonomía nos permite cargar de valor nuestra vida bien podemos decir que ella misma es valiosa ${ }^{43}$.

El que la autonomía personal sea moralmente valiosa es una razón para que todos llevemos vidas autónomas. Pero no es posible hacer autónomos a otros, el ejercicio de la autonomía está sobre los hombros de cada quien. Claro que los demás, particularmente el Estado, pueden ayudar y estimular la adopción y el desarrollo de modos autónomos de vida. Pero su ayuda está limitada a asegurar las condiciones de la autonomía ${ }^{44}$.

De aquí que del valor de la autonomía se deriven ciertos deberes, principalmente en cabeza del Estado pero que también alcanzan a los demás individuos:

Ayudar a crear las capacidades internas requeridas para llevar una vida autónoma: capacidades cognitivas o reflexivas, volitivas, emocionales, físicas, etc.

Crear o asegurar un adecuado rango y variedad de opciones moralmente valiosas sobre las cuales sea posible a los individuos ejercer sus capacidades de elección.

Abstenerse de la coacción y la manipulación (condición de independencia).

Evitar la coacción y la manipulación por parte de terceros.

Los dos primeros deberes en cabeza del Estado justifican políticas activas y van en contra de lecturas liberales tipo Estado gendarme. El cuarto es un deber positivo (tendiente

\footnotetext{
39 RAZ (1986) pp. 369-373.

40 RAZ (1986) pp. 378-381.

41 RAZ (1986) pp. 373-377.

42 RAZ (1986) pp. 377-378.

43 RAZ (1986) p. 387.

44 RAZ (1986) p. 407.
} 
a justificar políticas penales, por ejemplo) pero destinado a lograr que los terceros se abstengan de violar la condición de independencia. ¿Cómo es que la coerción y la manipulación (a las que apunta esta condición) afectan la autonomía y cómo es que un espacio libre de ellas la resguarda? Raz entiende que el manipular o coaccionar a otro puede expresar desprecio o falta de respeto por su autonomía. Además, obstaculiza el desarrollo de las referidas capacidades internas y pueden degradar el rango y reducir la variedad de las opciones significativas y moralmente valiosas que los demás tienen a su disposición.

El tercer deber sí es, claramente, un deber de abstención y justifica un ámbito de autonomía como no interferencia o libertad negativa. Lo que queda claro es que la libertad negativa, la libertad de coerción ajena, es valiosa en tanto sirve a la autonomía personal. Ahora bien, la doctrina de la libertad negativa con base en la autonomía tiene, entiende Raz, límites claros.

Primero, mientras la autonomía requiere la disponibilidad de un adecuado rango de opciones valiosas y significativas, no requiere la presencia de ninguna opción en particular. Salvo que alguien haya adoptado una opción (en ese caso eliminarla frustraría sus proyectos y decisiones y sería, consecuentemente, un ataque a su autonomía) una persona o un gobierno pueden tomar medidas para excluirla del rango de opciones disponibles sin merma para la autonomía ${ }^{45}$.

Segundo, el deber de asegurar un adecuado rango de opciones no se extiende a las opciones moralmente malas o repugnantes. En términos de Raz: "Dado que la autonomía es valiosa solo si tiene por objeto lo bueno, no confiere ninguna razón para proveer ni para proteger opciones malas y disvaliosas. Sin duda la autonomía es ciega a la calidad de las opciones elegidas. Una persona es autónoma incluso si elige mal. La autonomía es incluso parcialmente ciega a la calidad de las opciones disponibles. La persona es autónoma... solo si persigue lo bueno tal como ella lo capta. [Ahora bien], solo puede ser autónoma si cree que tiene opciones valiosas entre las cuales elegir. Esto es consistente con que varias de las opciones sean de hecho malas. Pero... las malas opciones no contribuyen en nada al valor de la autonomía. De hecho, elegir autónomamente lo malo hace la propia vida peor que lo que sería comparativamente una vida no autónoma. Dado que nuestra preocupación por la autonomía es una preocupación por capacitar a la gente a llevar una vida buena esto implica que tenemos razón para asegurar una autonomía tal que pueda ser buena. Y proveer, preservar o proteger opciones malas no nos capacita para disfrutar de una autonomía valiosa" 46 .

Importa ahora determinar si esta doctrina implica un permiso al Estado para disuadir a las personas de elegir opciones autorreferentes malas. ¿Es el principio raziano de autonomía y su modo de delimitar el espacio de no intervención consistente con la sanción legal de la moral privada? Si el Estado puede eliminar coactivamente opciones inmorales, entonces este pareciera ser el caso. Sin embargo las cosas son más complejas. Según Raz hay que distinguir entre opciones inmorales autorreferentes susceptibles y no susceptibles de coerción legal. El criterio de distinción es si la opción inmoral en cuestión produce un

\footnotetext{
45 RAZ (1986) pp. 410-411.

46 RAZ (1986) p. 411.
} 
daño a la autonomía del propio agente. Raz, contra Mill, entiende que el daño que habilita la intervención no es solo el daño a los demás sino también el daño que uno se pueda infligir a sí mismo. "Daño" es daño a la autonomía; que la autonomía en cuestión sea la propia o la de los demás es irrelevante ${ }^{47}$. De hecho, Raz entiende que "el principio de libertad con base en la autonomía... provee los fundamentos morales al principio de daño" 48 . La intervención estatal con las acciones autorreferentes entonces está habilitada cuando se dan conjuntamente (en los términos que discutiremos más adelante) la inmoralidad y el daño a la autonomía del agente.

Es evidente que hay una conexión entre la autonomía y el principio de daño ${ }^{49}$. Tanto cuando afectamos la capacidad de elegir de las personas (cuando, por ejemplo, manipulemos o dañamos sus capacidades reflexivas, volitivas, físicas, etc.) como cuando eliminamos opciones que podrían haber elegido o frustramos la persecución y el logro de proyectos, fines e incluso medios que ya han adoptado (cuando privamos a alguien de su propiedad, por ejemplo, impidiéndole el goce de las posibilidades que ese bien abría), les hacemos daño al disminuir su autonomía. Pero también es claro que si autorizamos al Estado a intervenir cuando el agente va contra sí mismo, desdibujamos el sentido en que el principio de daño es un límite. Se requiere un alto esfuerzo teórico que otorgue precisión esa nueva demarcación.

Hay, sin embargo, un tipo de intervención que esta nueva formulación del principio tajantemente excluye: el uso de la coerción para desalentar la adopción de decisiones inmorales pero no dañosas a la propia autonomía.

Precisemos el sentido de la anterior afirmación. Primero hay que tener en cuenta que la persecución de lo moralmente repugnante no puede ser defendida de la interferencia coercitiva sobre la base de que el hecho de que sea una elección autónoma le otorga valor. Con razón, Raz entiende que no lo hace ${ }^{50}$. Lo que hace que las acciones inmorales pero no dañosas ni para sí mismos ni para los demás no puedan ser objeto de coerción estatal es, nuevamente, el valor de la autonomía. Primero, esas acciones no dañan la autonomía. Si hubiera una justificación para someterlas a coerción debería derivarse de otros valores. Segundo, al perseguirlas coercitivamente agraviamos la autonomía de la persona en cuestión. Por una parte la coerción viola la condición de independencia y expresa generalmente falta de respeto para con el individuo a ella sometido. Además, la coerción (sobre todo la ejercitada mediante sanciones penales pero también otras formas menos severas) es una invasión de la autonomía global e indiscriminada. Encarcelar una persona le impide la persecución de casi todos sus proyectos autónomos. No hay modo de interferir solo con las elecciones moralmente repugnantes, necesariamente se interferirá con otras opciones ${ }^{51}$.

¿Cómo debería redactarse el principio constitucional si la teoría de Raz fuera verdadera? He aquí una propuesta sumamente tentativa: En tanto no impliquen daño a la pro-

\footnotetext{
RAZ (1986) p. 413.

48 RAz (1986) p. 400.

49 En el mismo sentido NinO (2007) p. 205.

50 RAZ (1986) p. 418.

51 RAZ (1986) pp. 418-19.
} 


\section{pia autonomía, los deberes de las personas para consigo mismas no son jurídicamente exigibles.}

Suponiendo que esta formulación identificara adecuadamente el principio de no interferencia que estamos buscando, ¿cómo funcionaría? ¿Qué casos quedarían por él protegidos? ¿Qué consecuencias prácticas tendría su sanción jurídica o su adopción como la interpretación correcta de una norma como el art. 19 de la Constitución argentina? En virtud del principio de daño definido sobre la base del principio de autonomía resulta que hay claras opciones autorreferentes que son, sin embargo, pasibles de disuasión por vía de coerción. El sujeto puede ser coercitivamente disuadido de ciertas opciones inmorales autónomas si dichas opciones han de dañar su capacidad futura de decidir autónomamente. De aquí que, por ejemplo, no parezca excluida la posibilidad de castigar el consumo de ciertas drogas de probado efecto incapacitante. Pero testeemos el criterio de Raz con nuestros casos testigo.

Revisemos primero la legitimidad de la norma que impone la clausura de los prostíbulos en Córdoba. Bien puede ponerse en cuestión que la elección de la prostitución como modo de vida sea una opción moralmente rechazable. Pero supongamos, argüendo, que lo es. Raz nos dice que las condiciones de la autonomía se satisfacen en la medida en que las personas gozan de un adecuado rango de opciones. Pero ninguna opción en particular es requerida, menos las opciones malas. Pareciera entonces que el Estado está autorizado a encarar una política de sustitución de la prostitución por otros trabajos moralmente inobjetables: si facilitase a las prostitutas la obtención de trabajos dignos y suficientes para sostener un nivel de vida medio entonces no estaría infringiendo su autonomía. Lo que no puede hacer el Estado es prohibir y sancionar coercitivamente el ejercicio de la prostitución. Pues la prostitución, aun si inmoral, no es dañosa ni para los terceros ni para la prostituta. No lo es en el sentido relevante de daño: privación de un adecuado rango y variedad de opciones, de la habilidad de usarlas y frustración de la persecución y el logro de los proyectos en que el agente ya está embarcado. Claramente al cerrar sus lugares de trabajo se les generan a las prostitutas obstáculos que dificultan el logro de sus fines y proyectos autónomamente elegidos. De ser correcta la teoría de Raz, la prohibición en cuestión resultaría moralmente ilegítima. De sancionarse constitucionalmente un principio como el propuesto, dicha prohibición devendría inconstitucional. En el caso del derecho argentino, tal sería la calificación de la ley de trata si esta fuera la lectura correcta del artículo 19 de su Constitución.

Respecto de la penalización del consumo de prostitución adulta y consentida sucede más o menos lo mismo. Pues el uso de la coerción solo está permitido cuando se daña a otros o a uno mismo. Por más inmoral que nos pueda parecer la utilización del sexo de otra persona como bien de uso disponible en el mercado, no parece que se satisfagan las condiciones expuestas y por lo tanto desde la teoría de Raz la coerción no está justificada para estos casos.

Toca ahora revisar los problemas de la teoría. Lo hagamos analizando la relación entre daño a la propia autonomía e inmoralidad. Un primer modo concebirla es entender que estas condiciones son independientes, que lo que hace inmoral un acto es distinto de lo que lo hace dañoso a la autonomía. Si esta es la comprensión correcta, toda acción inmoral pero no dañosa o dañosa pero no inmoral está fuera del alcance de la coerción legítima. Un 
segundo modo es pensar que ambas categorías están conceptual o normativamente vinculadas. Por ejemplo (y esta entiendo que es la postura de Raz) que la autonomía es un valor moral y, por consiguiente, que todo daño a la autonomía es inmoral. Bajo esta concepción la clase de las acciones dañosas pertenecería enteramente a la clase de las acciones inmorales. Claro que también podría haber actos inmorales para con uno mismo que lo fueran aun sin dañar la autonomía (estos serían inatacables).

En el primer caso la doble exigencia funciona como una garantía. Acciones (bajo cierta concepción) inmorales, como la prostitución, el consumo de pornografía, etc., pueden aun no dañar la autonomía y por lo tanto estar exentas de la autoridad de los magistrados. Lo mismo pasaría con acciones dañosas a la autonomía pero no inmorales. El problema es que para que esta concepción funcione debemos contar con una teoría moral (seguramente controvertida) que nos diga qué actos autorreferentes son inmorales.

En todo caso no es esta la concepción de Raz. Él afirma que hay una conexión conceptual entre daño e inmoralidad: "En tanto que 'causar daño' implica por su propio significado que la acción es, prima facie, mala, es un concepto normativo que adquiere su significado específico de la teoría moral dentro de la que está incrustado. Sin una conexión de este tipo a una teoría moral el principio del daño es un principio formal carente de contenido concreto y específico, que no conduce a ninguna política" ${ }^{52}$.

La idea de Raz, como dije arriba, parece ser que toda acción autorreferente dañosa para la propia autonomía es por esa razón inmoral. Pero esto o bien es absurdo o bien hace que la idea de daño deje de funcionar como un límite claro a la intervención. Veamos.

Recordemos que el daño a la autonomía está dado básicamente por la eliminación de opciones y la restricción de capacidades. Analicemos la primera. ¿Está justificada la intervención en cualquier acto autorreferente que elimine opciones a futuro? La respuesta es manifiestamente negativa. Como el mismo Raz reconoce ${ }^{53}$ cualquier decisión de un sujeto autónomo implica que ciertas opciones ya no están disponibles (el matrimonio no es un ejemplo menor). Por lo tanto cualquier decisión implicaría daño a sí mismo y habilitaría la intervención, lo cual es absurdo ${ }^{54}$. Hay que dar cuenta entonces del criterio en virtud del cual ciertas eliminaciones de opciones habilitan la intervención y ciertas no. En todo caso este criterio externo a la mera eliminación de opciones será un criterio moral, con lo cual volveríamos a la alternativa arriba analizada (dos condiciones separadas).

Quizás lo que determine la inmoralidad de los actos autorreferentes no sea la eliminación de opciones sino la restricción de capacidades (cognitivas, volitivas, etc.). Bajo esta opción la inmoralidad de un acto autorreferente se reduciría a su carácter dañoso a la autonomía en virtud de restringir las capacidades del propio agente. La idea (aunque en un sentido no reduccionista) tiene una noble tradición. En Kant, por ejemplo, buscar la propia perfección, entendida como el cultivo de todas las facultades corporales y espirituales en tanto pueden sernos útiles para toda suerte de fines posibles (siendo la capacidad

\footnotetext{
52 RAZ (1986), p. 414.

53 RAZ (1986) p. 374, nota 1.

54 Agradezco a Rodrigo Correa González y a Moisés Vaca el haberme señalado la importancia de este problema.
} 
de adoptar fines lo específico de la humanidad), es un deber para con uno mismo ${ }^{55}$. De ser este el criterio podrían exigirse coactivamente todas aquellas acciones necesarias para el desarrollo de nuestras facultades y todas aquellas omisiones requeridas para evitar su degradación. Esto sería compatible con políticas dirigidas a perseguir el consumo de drogas severamente incapacitantes, por ejemplo. Pero también con la persecución de vicios menos gravosos como el hábito de fumar, pues el fumar orada la voluntad. Y no solo eso sino también conductas indolentes que dejan de cultivar las propias facultades. Pero no calza con nuestras intuiciones el que se pueda exigir coactivamente el desarrollo de nuestras propias facultades, al menos no en adultos. En cuanto a las omisiones, su exigencia coactiva no sería sino crudo perfeccionismo, justamente una de las doctrinas contra la que dio batalla el principio de daño en su formulación canónica, liberal. Sin dudas Raz estará conforme con estos resultados; no es obvio que lo estemos nosotros.

\section{A MODO DE CONCLUSIÓN}

Tal como sostuve más arriba, ofrecer una evaluación acabada de las credenciales de las teorías expuestas, tanto en el ámbito conceptual como en el normativo, está más allá de las posibilidades del presente trabajo. En todo caso creo poder afirmar lo siguiente. En primer lugar, las concepciones perfeccionistas puras valoran insuficientemente la libertad de las personas de elegir el modo en que han de vivir sus propias vidas. Esto es incompatible con la forma y los valores básicos de sociedades pluralistas como las nuestras. Hay también buenas razones, me parece, para rechazar un liberalismo radical tal que exija el respeto de cualquier plan de vida con total independencia de su contenido. Dicha teoría parece depender de una concepción empírica de las metapreferencias: lo único que marcaría un plan de vida como valioso y digno de respeto es el hecho de que la persona en cuestión se identifique con él. Pero ante esto tenemos dos dificultades: por un lado la identificación sería equivalente a la coincidencia entre los deseos de segundo y primer orden. Pero una coincidencia fáctica no puede dar cuenta de por qué hemos de atribuir valor y respetabilidad (blindaje contra la coerción) a los planes de vida (de un hecho no se derivan valores). Además, la concepción empírica no permite dar cuenta de valor que atribuimos al modo en que hemos llegado a tener los planes de vida que tenemos. Nos importa no solo que sean nuestros sino que sean producto de una elección consciente, mínimamente reflexiva. Si esto es así los planes de vida que pongan en peligro nuestras capacidades reflexivas a futuro bien pueden ser cuestionados: el Estado tendría un derecho moral a desalentarlos incluso coercitivamente. La indagación parecería indicar entonces la plausibilidad de una fundamentación de los límites de la interferencia estatal en una teoría de la autonomía personal como la de Raz. Claro que, para ello, una teoría semejante debería dar muchas respuestas. En última instancia una doctrina que limite coactivamente acciones que ponen en cuestión nuestras facultades no es otra cosa que una doctrina perfeccionista. Pero nos creemos con derecho a disponer sobre nuestras propias facultades, por lo menos en algún grado. La teoría entonces debería delimitar claramente el ámbito de las intervenciones perfeccionistas

55 KANT, VI, 387, 444. 
justificadas. Hasta que estas respuestas sean dadas, y pese a las dificultades en su momento desarrolladas, tal vez la mejor opción sea atenernos a la formulación amplia y abstracta del principio de libertad negativa.

\section{BIBLIOGRAFÍA CITADA}

Atria, Fernando (2012): "Lo que importa sobre los principios", El Cronista del Estado Social y Democrático de Derecho, No. 25, 2012: pp. 72-83.

Atria, Fernando, (2016): La Forma del Derecho (Madrid, Marcial Pons)

BaYÓn Mohíno, Juan Carlos (1996): "Principios y reglas: legislación y jurisdicción en el Estado constitucional”, Jueces para la democracia, No 27: pp. 41-49.

Christman, John (2011): "Autonomy in Moral and Political Philosophy", The Stanford Encyclopedia of Philosophy (Spring Edition), ZaLTA, Edward N. (ed.), URL = http://plato. stanford.edu/archives/spr2011/entries/autonomy-moral/

Devlin, Patrick (1965): The Enforcement of Morals (Oxford, Oxford University Press). Hay versión castellana en Devlin, Patrick (2010): La Imposición de la Moral (traducción de Miguel Ángel Ramiro, Andrea Rodríguez Liboreiro y Marta Sosa, Madrid, Dykinson por donde se cita).

Dworkin, Gerald, (1976): "Autonomy and Behavior Control", en The Hastings Center Report, Vol. 6, № 1: pp. 23-28.

Dworkin, Gerald, (1988): Theory and Practice of Autonomy (New York, Cambridge University Press).

Dworkin, Ronald, (1993): Los Derechos en Serio, trad. de Marta Guastavino (Buenos Aires, Planeta Agostini).

Dworkin, Ronald, (1986): Law's Empire (Cambridge, Harvard University Press).

Dworkin, Ronald (2012): “¿Tenemos Derecho a la Pornografía?” en Dworkin, Ronald Una Cuestión de Principios (trad. de Victoria Boschiroli, Buenos Aires, Siglo Veintiuno) pp. 411-456.

FinNis, John (1987): "Legal Enforcement of 'Duties to Oneself': Kant v. Neo-Kantians", Columbia Law Review, Vol. 87: pp. 433-456.

FeinberG, Joel (1986): Harm to Self (New York, Oxford University Press).

Frankfurt, Harry (1971): "Freedom of the Will and the Concept of a Person", The Journal of Philosophy, Vol. 68, No. 1: pp. 5-20.

García Pino, Gonzalo y Contreras Vásquez, Pablo (2014): Diccionario Constitucional Chileno Cuadernos del Tribunal Constitucional.

Gaus, Gerald (2011): "Liberalism”, The Stanford Encyclopedia of Philosophy, Edward N. ZALTA (ed.); URL = http://plato.stanford.edu/archives/spr2011/entries/liberalism/

HAKSAR, Vinit (1979): Equality, Liberty and Perfectionism (New York, Oxford University Press).

Hart, Herbert (1963): Law, Liberty and Morality (Oxford, Oxford University Press). Hay versión castellana en HaRT, Herbert (2006): Derecho, Libertad, Moralidad (traducción de Miguel Ángel Ramiro Avilés, Madrid, Dykinson) por donde se cita.

Hurka, Thomas (1993): Perfectionism (Nueva York, Oxford University Press). 
IosA, Juan — Libertad negativa, autonomía personal y Constitución..

Kant, Inmanuel, (1996-VI-): Metafísica de las Costumbres (trad. de Adela Cortina y Jesús Conill Sancho, Barcelona, Altaya).

Kant, Inmanuel (2004-VIII): “Teoría y Práctica”, en ¿Qué es la Ilustración? (trad. de Roberto Aramayo, Madrid, Alianza).

LAPORTA, Francisco (2007): El Imperio de la Ley, una visión actual (Madrid, Trotta).

MiLl, John (1984): Sobre la Libertad (Madrid, Sarpe).

Nino, Carlos (2007) Ética y Derechos Humanos (Buenos Aires, Astrea).

Quong, Jonathan (2011): Liberalism without Perfection (New York, Oxford University Press).

Rawls, John (1971): A Theory of Justice (Cambridge, Cambridge University Press).

Raz, Joseph (1986): The Morality of Freedom (Oxford, Clarendon Press).

Raz, Joseph (1991): Razón Práctica y Normas (Madrid, Centro de Estudios Constitucionales).

Stephen, James (1991): Liberty, Equality, Fraternity (Chicago, University of Chicago Press).

Valiente Noailles, Carlos (1966): La Moral Pública y las Garantías Constitucionales (Buenos Aires, La Ley).

Wall, Stephen (1998): Liberalism, Perfectionism and Restraint (Cambridge, Cambridge University Press).

Wall, Stephen (2012): "Perfectionism in Moral and Political Philosophy", The Stanford Encyclopedia of Philosophy, ZaLTA, Edward N. (ed.); URL = https:// plato.stanford.edu/entries/perfectionism-moral/\#PerValThe 